\title{
Wer hat die Wahl? Robin Markwicas ,Emotional Choices. How the Logic of Affect Shapes Coercive Diplomacy“ - Eine Reflexion
}

\author{
Anna Rauscher $\cdot$ Jessica Gienow-Hecht
}

Online publiziert: 31. Juli 2020

(C) Der/die Autor(en) 2020, korrigierte Publikation 2022

Zusammenfassung Robin Markwicas Monografie erforscht, welche Rolle Emotionen in der Geschichte von (außen-)politischen Prozessen und Beziehungen spielen können. Entsprechend setzen die Autorinnen der Rezension Markwicas Arbeit in Beziehung zur Emotionsgeschichte als Forschungsparadigma. Zudem referieren sie anhand ausgewählter Arbeiten, welchen Erkenntnisgewinn eine Berücksichtigung der Emotionen in der Geschichte der internationalen Beziehungen liefert. Im Anschluss daran evaluieren sie die theoretischen und methodischen Überlegungen des Autors, die Ergebnisse seiner Analyse und seine Vorschläge zur konstruktiven Anwendung dieser Resultate in der internationalen Diplomatie. Die Autorinnen kommen zu dem Schluss, dass Markwicas Ansatz aufgrund seines Fokus auf Emotion als eine weitere Variable in (außen-)politischen Prozessen, sowie wegen der relativen Offenheit, Pluralität und Flexibilität des Analysemodells die Diskussion um Entscheidungsfindungzprozesse nachhaltig inspirieren könne.

Schlüsselwörter Emotionsgeschichte · US-Außenpolitik · Diplomatiegeschichte · Geschichte der internationalen Beziehungen · Neuere Geschichte

\footnotetext{
A. Rauscher $(\bowtie) \cdot$ J. Gienow-Hecht

John-F.-Kennedy-Institut für Nordamerikastudien, Freie Universität Berlin, Berlin, Deutschland

E-Mail: rauschea@zedat.fu-berlin.de

J. Gienow-Hecht

E-Mail: j.gienow@fu-berlin.de
} 


\title{
Whose Choice Is It? Robin Markwica's "Emotional Choices. How the Logic of Affect Shapes Coercive Diplomacy"-A Reflection
}

\begin{abstract}
This review places Robin Markwica's book, "Emotional Choices" in the context of two recently emerging research fields: the history of emotions and the role of emotions in international relations. Markwica argues that individual identity, social norms and emotions impact decisions in situations of crisis, then weaves these findings into an "emotional choice theory". He applies his theory to US actions and perceptions during the Cuban Missile Crisis and the Second Gulf War, concluding that emotions provide a helpful category to define rules of action in international coercive diplomacy.
\end{abstract}

Keywords History of Emotions · US Foreign Policy · Diplomatic History · History of International Relations · Modern History

Im Sommer 1952 sandte der US-amerikanische Botschafter in Teheran, Loy Henderson, eine Serie von Telegrammen nach Washington, die die mentale Zurechnungsfähigkeit des iranischen Premierministers ernsthaft infrage stellten. Mohammed Mossadeq, so Henderson, sei nicht in der Lage ,to carry on complicated negotiations for any length of time in a single direction." Schlimmer noch, statt „rational“ zu denken, betrachte er ,,international politics from [an] emotional point of view“, während seine Anhänger - allesamt „mad and suicidal“ - ihm wie irregeleitete Lemminge blind folgten. ${ }^{1}$ Was war passiert? Mossadeq hatte den USamerikanischen Botschafter in einem pyjamaähnlichen Gewand auf einem Diwan liegend empfangen und während der Verhandlungen über die Nationalisierung der iranischen Ölquellen verschiedentlich geweint und geschrien. Solch ein Benehmen war in Hendersons Weltbild eines Staatsmannes unwürdig: Ein Premier weine nicht und zeige keine Emotion. ${ }^{2}$ Henderson war mit seiner Haltung dabei keineswegs allein, wie Mary-Ann Heiss gezeigt hat: In den Akten des US-amerikanischen und des britischen Außenministeriums finden sich eine Vielzahl von mehr oder minder verächtlichen Aussagen, die den iranischen Führer mit Attributen wie ,irre“, ,emotional“, „krank“, „,dement“, „hysterisch“, ,,neurotisch“" und vielen anderen Dingen mehr beschreiben. ${ }^{3}$ Indes hatte Mossadeq genau das getan, was viele Politiker im Mittleren Osten taten: Er zeigte als Teil seines Amtes Emotion, um ein politisches Argument zu unterstreichen. Beide Männer, Henderson und Mossadeq, hatten völlig unterschiedliche kulturelle Präkonzeptionen über akzeptable und inakzeptable Verhaltensweisen im politischen Prozess und darüber, welches Benehmen ein Staatsmann im Dienst zeigt. US-amerikanische Politiker misstrauten Mossadeq, weil er

\footnotetext{
${ }^{1}$ Heiss, Mary-Ann: Real Men Don't Wear Pajamas. Anglo-American Cultural Perceptions of Mohammed Mossadeq and the Iranian Oil Nationalization Dispute, in: dies./Hahn, Peter (Hrsg.): Empire and Revolution. The United States and the Third World Since 1945, Ohio State UP, Columbus 2001, S. 178-194, hier S. $185,187$.

2 Dies.: Cultural Perceptions (wie Anm. 1), S. $183 \mathrm{f}$.

3 Ebd., S. 184, 186.
} 
sich, wie sie sagten, ,weiblich“ betrug. Und genau diese Voreingenommenheit hielt sie davon ab, zu hören, was er eigentlich sagte. Diese miteinander im Konflikt stehenden Wahrnehmungen kollidierten in den Gesprächen zur Ölfrage, führten zur Einstellung jeglicher US-Unterstützung für Mossadeq, später zum Coup von 1953 mit den bekannten Folgen. ${ }^{4}$

Was wäre nun passiert, wenn sich der US-amerikanische Botschafter und der iranische Premier während der Verhandlungen in Teheran einem Coaching gestellt hätten? Hätte der Disput durch die Selbstreflexion über die eigene Emotion anders, vielleicht sogar besser gelöst werden können? Was wäre passiert, wenn sich Nikita Chruschtschow und Saddam Hussein auf die Couch gelegt hätten? Welchen Unterschied machen Gefühle im politischen Entscheidungsprozess? Was können wir von der Analyse von Emotionen für zukünftige Krisenszenarien lernen?

Mit diesen Fragen beschäftigt sich der Politikwissenschaftler Robin Markwica in der vorliegenden, seiner ersten Monografie. Markwica ist Max Weber Fellow am Robert Schuman Centre for Advanced Studies am European University Institute (Fiesole) und Research Associate am Centre for International Studies der Universität Oxford. Seine Forschungsinteressen umfassen die Theorie der internationalen Beziehungen, internationale Sicherheit, Krieg und Frieden, und die Analyse von Außenpolitik. Weitere Schwerpunkte sind Emotionsforschung und konstruktivistische und psychologische Ansätze zur Erforschung internationaler Beziehungen. ${ }^{5}$

Markwicas Monografie „Emotional Choices“ basiert auf der Prämisse, dass Analytiker von politischen Verhaltens- und Entscheidungsfindungsprozessen sich zwar ausführlich mit rationaler Entscheidungstheorie und normativen Modellen auseinandergesetzt haben, durchaus auch Interpretationen der Soziologie und der Kulturwissenschaften im Sinne von Reflexion, Struktur, Gruppenverhalten und Umwelt zulassen, dabei aber eine dritte Kategorie außer Acht lassen, nämlich die der Emotion. Markwica legt in seinem Buch ein Handlungsmodell vor, um erklärbar zu machen, weshalb und unter welchen Bedingungen Staatsmänner sich diplomatischen Zwangsandrohungen eines mächtigeren Kontrahenten widersetzen - oder eben einlenken. Dabei beruhen die Überlegungen des Autors auf der Grundannahme, dass Emotionen eine vernachlässigte Schlüsselrolle in außenpolitischen Fragen spielen. Essenziell, so konstatiert der Autor, sind Emotionen auch bezüglich möglicher Reaktionen von Politikern in außenpolitischen Bedrohungssituationen, da Gefühle das Entscheidungsverhalten auf vorhersehbare Weise beeinflussen. Diesen Voraussetzungen entsprechend entwickelt Markwica eine Theorie emotionaler Entscheidungen (,emotional choice theory“) für die internationalen Beziehungen.

Damit widmet sich Markwica einer Thematik, die seit etwa einer Dekade zunehmend an Konjunktur gewinnt, nämlich der Frage nach der Rolle von Emotionen in der Geschichte von (außen-)politischen Prozessen und Beziehungen. Nach einem knappen Überblick über die Emotionsgeschichte als Forschungsparadigma referieren wir anhand ausgewählter Arbeiten, welchen Erkenntnisgewinn eine Be-

\footnotetext{
${ }^{4}$ Ebd., S. 181, 183 f., 187 f., $190 \mathrm{f}$.

${ }^{5}$ Siehe auch die biografischen Angaben zu Markwica auf der Seite des European University Institute, URL: <https://www.eui.eu/ProgrammesAndFellowships/MaxWeberProgramme/People/MaxWeberFellows/ Fellows-2017-2018/MARKWICA> [Zugriff: 22.02.2019].
} 
rücksichtigung der Emotionen in der Geschichte der internationalen Beziehungen liefert. Darauf folgt eine Darstellung und Evaluation der von Markwica vorgelegten theoretischen und methodischen Überlegungen, seiner Analyseergebnisse und seiner Empfehlungen, wie sich diese Resultate in der internationalen Diplomatie konstruktiv anwenden lassen.

\section{Konjunktur der Gefühle}

Seit den 1990er Jahren ist in der Geschichtswissenschaft ein rasch wachsendes Interesse an Emotionen zu verzeichnen, sodass zuweilen von einem emotional turn die Rede ist. ${ }^{6}$ Wegbereitend war dafür seit den 1970er Jahren eine Reihe von Faktoren, darunter die prominente Rolle von Gefühlen im kulturellen Klima des letzten Viertels des 20. Jahrhunderts; kulturtheoretische Erkenntnisse im Bereich der postkolonialen Theorie; anthropologische Be- und Zuschreibungen von Emotionen, die zwischen westlichen und nicht-westlichen Emotionswelten unterschieden; sowie neurowissenschaftliche Forschungen, die die Dichotomie zwischen Gefühlen und rationalem Denken infrage stellten. ${ }^{7}$ Während frühe Vertreter wie Norbert Elias Emotionsgeschichte als linear darstellten, setzten sich, angefangen mit der Arbeit Lucien Febvres, anti-teleologische und nicht-lineare Ansätze durch. ${ }^{8}$

Unter Historiker_innen herrscht weitgehend Konsens darüber, dass nur die Manifestationen von Gefühlen, weniger jedoch tatsächlich empfundene Emotionen Gegenstand der Geschichtsschreibung sein können. ${ }^{9}$ Um erstere zu erforschen, liegt

\footnotetext{
6 Zum Begriff des emotional turn siehe Plamper, Jan: The History of Emotions. An Interview with William Reddy, Barbara Rosenwein, and Peter Stearns, in: History and Theory 49 (2010), H. 2, S. 237-265, hier S. 237. Zur Konjunktur der Emotionsgeschichte seit den 1990er Jahren und zur allgemeinen Einführung in das Feld siehe Matt, Susan J.: Current Emotion Research in History. Or, Doing History from the Inside Out, in: Emotion Review 3 (2011), H. 1, S. 117-124, hier S. 118; Überblicksdarstellungen finden sich auch in Hitzer, Bettina: Emotionsgeschichte. Ein Anfang mit Folgen, in: H-Soz-Kult. Kommunikation und Fachinformation für die Geschichtswissenschaften, 23. November 2011, URL: <https://www.hsozkult. de/literaturereview/id/forschungsberichte-1221> [Zugriff: 18.02.2019]; Plamper, Jan: Geschichte und Gefühl. Grundlagen der Emotionsgeschichte, Siedler, München 2012; Frevert, Ute: History of Emotions, in: Feldman Barrett, Lisa/Lewis, Michael/Haviland-Jones, Jeanette (Hrsg.): Handbook of Emotions, Guilford, New York ${ }^{4} 2016$, S. 49-65; Rosenwein, Barbara H./Cristiani, Riccardo: What Is the History of Emotions? Polity Press, Cambridge 2018; Boddice, Rob: The History of Emotions, Manchester UP, Manchester/New York 2018.

7 Frevert: Emotions (wie Anm. 6), S. 62.

8 Ebd., S. 59-62. Die Autorin nimmt hier Bezug auf Elias, Norbert: Über den Prozess der Zivilisation. Soziogenetische und psychogenetische Untersuchungen, Haus zum Falken, Basel 1939; Febvre, Lucien: Sensibility and History. How to Reconstitute the Emotional Life of the Past, in: ders.: A New Kind of History and Other Essays, hrsg. v. Peter Burke, übers. v. Keith Folca, Harper and Row, New York u.a. 1973, S. 12-26 und ders.: Honneur et Patrie, Perrin, Paris 1996. Eine Kritik an teleologischen Ansätzen formuliert Rosenwein, Barbara H: Worrying About Emotions in History, in: American Historical Review 107 (2002), H. 3, S. 821-845, hier S. 827; und dies.: Problems and Methods in the History of Emotions, in: Passions in Context. International Journal for the History and Theory of Emotions 1 (2010), H. 1, S. 1-33, hier S. 21, URL: <https://www.passionsincontext.de/uploads/media/01_Rosenwein.pdf> [Zugriff: 12.04.2019].

9 Im Gegensatz zu Peter N. und Carol Z. Stearns, die in: Emotionology. Clarifying the History of Emotions and Emotional Standards, in: American Historical Review 90 (1985), S. 813-836, hier S. 825, 830, davon
} 
mittlerweile eine Vielzahl an theoretischen Ansätzen vor. Carol Z. und Peter N. Stearns beschreiben mit dem Konzept der emotionology die Normen und Haltungen, die eine soziale Gruppe mit Emotionen verbindet. Diese unterschieden sich je nach sozialer Gruppe und seien historischem Wandel unterworfen, weshalb sie, so die Autor_innen, andere soziale Veränderungsprozesse erklären könnten. ${ }^{10}$ Erklärungspotenzial für den Wandel von Gesellschaftssystemen schreibt auch William Reddy den Emotionen zu und entwickelt dafür das Konzept der emotional regimes. ${ }^{11} \mathrm{Um}$ das Zusammenspiel von Emotion und Ethik unter Eliten im Alten Rom zu erklären, spricht Robert A. Kaster von emotional scripts. Er versteht Emotionen als narrativen Prozess und sieht darin eine Möglichkeit, die Schwierigkeit zu umgehen, Emotionen wiederum nur mit alternativen Emotionsbegriffen zu beschreiben. ${ }^{12}$ Die Mediävistin Barbara H. Rosenwein entwickelt eine Theorie der emotional communities, die kongruent mit anderen sozialen Gruppierungen und von Emotions-Systemen geprägt seien, wobei Individuen verschiedenen emotionalen Gemeinschaften angehörten. ${ }^{13}$ Bettina Hitzer schließlich bezweifelt den epistemischen Mehrwert dieser Konzeptualisierung und fragt stattdessen, ob „emotionale Gemeinschaften“ Verbindungen knüpfen können, die „quer zu sozialen oder politischen Gruppierungen“ verlaufen. ${ }^{14}$

Um die Manifestationen vergangener Emotionen zu erforschen, schlagen die genannten Autor_innen eine Fülle an Strategien zum close reading textueller Quellen vor. ${ }^{15}$ Auch Gestik, andere nonverbale Äußerungen und Auslassungen seien zu beachten. ${ }^{16}$ Emotionsgeschichte sei, so Frevert, als eine Geschichte von Praktiken zu denken, da nur soziale Praktiken Emotionen überhaupt erst mit Bedeutung ausstatteten. Entsprechend müssten Historiker_innen mit den jeweiligen sozialen, ökonomischen, politischen und kulturellen Kontexten vertraut sein und Emotionen im Kontext der Praktiken betrachten, zu denen sie gehören. ${ }^{17}$ Beim Erforschen vergangener Gefühle anderer sollte schließlich, so merken einige Autor_innen an, auch das eigene Gefühlsleben mitbedacht werden. Während einige vor einer unkritischen Übertragung der eigenen Auffassung von Emotionen auf andere historische und kulturelle Situationen warnen, versuchen andere, das Potenzial der eigenen Emo-

ausgehen, dass vergangene emotionale Erfahrungen erforschbar seien, betont Rosenwein: Problems and Methods (wie Anm. 8), S. 11, Anm. 37, dass lediglich erforschbar sei, wie historische Akteure Gefühle artikulierten, verstanden und repräsentierten. Dass Historiker_innen Emotionen nur in mediatisierter Form untersuchen könnten, betonen auch Kaster, Robert A.: Emotion, Restraint, and Community in Ancient Rome, Oxford UP, Oxford u. a. 2005, S. 10 und Costigliola, Frank: Reading for Emotion, in: ders./Hogan, Michael J. (Hrsg.): Explaining the History of American Foreign Relations, Cambridge UP, Cambridge ${ }^{3} 2016$, S. 356-374, hier S. 358.

10 Stearns/Stearns: Emotionology (wie Anm. 9), S. $813 \mathrm{f} ., 828 \mathrm{f}$.

${ }^{11}$ Reddy, William M.: The Navigation of Feeling. A Framework for the History of Emotions, Cambridge UP, Cambridge 2001, S. 315, 323.

${ }^{12}$ Kaster: Emotion, Restraint, and Community (wie Anm. 9), S. 3, 8, 11.

13 Rosenwein: Emotions (wie Anm. 8), S. 842.

${ }^{14}$ Hitzer: Emotionsgeschichte (wie Anm. 6), S. 10.

15 Rosenwein: Problems (wie Anm. 8), S. 12-21.

16 Gould, Deborah Bejosa: Moving Politics. Emotion and Act Up's Fight Against Aids, Chicago UP, Chicago, IL 2009, S. $29 \mathrm{f}$.

17 Frevert: Emotions (wie Anm. 6), S. 53-57. 
tionen für den Erkenntnisprozess aufzuzeigen. ${ }^{18}$ Für Deborah Bejosa Gould etwa sind Introspektion und emotionales Wissen eine zentrale Ressource, um schriftliche und mündliche Quellen auf Affekte hin zu analysieren. Benno Gammerl fordert im Kontext eines Oral-History-Projekts, die Funktion der eigenen Emotionen im Forschungsprozess zu reflektieren, um verkürzende Darstellungen zu vermeiden und das Erkenntnispotenzial der eigenen Gefühle offenzulegen. ${ }^{19}$

Markwica plädiert bezüglich der Subjektivität des Forschers für einen Kompromiss. Im Idealfall, so Markwica, sollten die Bedeutungen von Emotionen der forschenden Person zwar vertraut, aber auch losgelöst vom eigenen kulturellen Hintergrund zu betrachten sein. Optimale Ergebnisse ließen sich erzielen, wenn die lokalen Emotionsnormen und affektiven Konnotationen von Wörtern, wie etwa Ironie oder Sarkasmus, zwar verstanden, jedoch zugleich nicht derart internalisiert würden, dass sie gar nicht mehr wahrgenommen würden.

In welchem Ausmaß die Emotionsgeschichte und die Emotionsforschung mittlerweile an Bedeutung gewonnen haben, zeigen eine Fülle an thematisch relevanten Publikationen, Tagungen und die akademische Institutionalisierung des Feldes in Form von Forschungszentren und -projekten. Sie lassen sich an dieser Stelle lediglich in einer Auswahl darstellen: Federführend muss hier die Emotionshistorikerin Ute Frevert genannt werden, die den seit 2008 am Max-Planck-Institut für Bildungsforschung bestehenden Bereich „Geschichte der Gefühle“ leitet. Der Bereich untersucht unter Hinzuziehung anderer Disziplinen die emotionalen Codes, Regimes und Lexika von Gesellschaften. Sein zeitlicher Fokus liegt primär auf dem Zeitraum vom 18. bis 20. Jahrhundert; geografisch konzentriert er sich auf den europäischwestlichen und südasiatischen Raum. Das „Australian Research Council Centre of Excellence for the History of Emotions" nahm 2011 seine Arbeit auf und hat seinen anfänglichen Fokus auf europäische Gesellschaften im Zeitraum 1100 bis 1800 mittlerweile beträchtlich erweitert. Zu Emotionen in der Frühen Neuzeit und Moderne arbeitet unter anderem Claudia Jarzebowski am Friedrich-Meinecke-Institut der Freien Universität Berlin, wo sie zum Beispiel Konferenzen zu Emotionen und Kulturkontakt vor 1800 (2016) und zum Verhältnis von Politik und Emotion in der Vormoderne (2011) initiiert hat. ${ }^{20}$ Arbeiten zur Emotionsgeschichte haben in jüngerer Zeit Barbara Rosenwein (Loyola University Chicago), Piroska Nagy (Université

\footnotetext{
18 Dass ein unkritisches Übertragen der Emotionskonzepte der eigenen Kultur auf den Untersuchungsgegenstand den Erkenntniswert einschränken kann, merken u.a. Stearns/Stearns: Emotionology (wie Anm. 9), S. 823, an. Zum Potenzial der eigenen Emotionen im Forschungsprozess siehe Gammerl, Benno: Can You Feel Your Research Results? How to Deal with and Gain Insights from Emotions Generated During Oral History Interviews, in: Flam, Helena/Kleres, Jochen (Hrsg.): Methods of Exploring Emotions, Routledge, London/New York 2015, S. 153-162 und Gould, Deborah Bejosa: When Your Data Make You Cry, in: ebd., S. 163-171.

19 Gould: Data (wie Anm. 18), S. 163, 168 ff.; Gammerl: Results (wie Anm. 18), S. 153 f., 160.

20 Eine Einführung zum Forschungsbereich „Geschichte der Gefühle“ am Max-Planck-Institut für Bildungsforschung sowie die einzelnen Projekte finden sich unter URL: <https:/www.mpib-berlin.mpg.de/ de/forschung/geschichte-der-gefuehle> [Zugriff: 22.02.2019]; näheres zum „Australian Research Council Centre of Excellence for the History of Emotions“" unter URL: <http://www.historyofemotions.org. au/> [Zugriff: 22.02.2019]; Details zum interdisziplinären Symposium „Performing Emotions! Zum Verhältnis von Politik und Emotion in der frühen Neuzeit und in der Moderne" unter URL: <https:// www.geschkult.fu-berlin.de/e/fmi/institut/arbeitsbereiche/ab_jarzebowski/media/Performing_Emotion_ Flyer.pdf> [Zugriff: 22.02.2019]; zur Konferenz „Emotions-Movement, Cultural Contact and Exchange,
} 
du Québec à Montréal), Damien Bouquet (Aix-Marseille-Université), Gerd Althoff (Westfälische Wilhelms-Universität Münster), Claudia Jarzebowski (Freie Universität Berlin) und Rob Boddice (Freie Universität Berlin) vorgelegt. ${ }^{21}$ Hinzu treten zahlreiche Konferenzen zum Thema Emotion, Gesellschaft und Politik in jüngster Zeit, wie zum Beispiel die von Barbara Keys und Jessica Gienow-Hecht ausgerichtete Tagung zum Thema „Emotions and International Relations“ (Melbourne 2014). Formen affektiver Kollektivität waren Thema einer von der Theaterwissenschaftlerin Doris Kolesch und dem Soziologen Hubert Knoblauch 2018 am Zentrum „Affective Societies. Dynamics of Social Coexistence in Mobile Worlds“ (Freie Universität Berlin) organisierten Tagung mit dem Titel „Public Emotions. Affective Collectivity in Audiences“. Mit „Emotionen, Politik und Medien in der Zeitgeschichte“ befasste sich im Oktober 2018 eine Konferenz an der Universität des Saarlandes und ein von Hélène Miard-Delacroix und Andreas Wirsching organisiertes Kolloquium mit dem Titel „Internationale Beziehungen und ,emotional regimes“. Neue Fragen an die Geschichte des Kalten Krieges" am Historischen Kolleg München im gleichen Jahr. Emotionen spielen schließlich in dem am John-F.-Kennedy-Institut der Freien Universität Berlin angesiedelten Forschungsprojekt „The Quest for Harmony: Music, Emotion, and the Discourse of Human Rights in the United States Since World War Two" eine zentrale Rolle. Und dass Emotionen nicht nur für Historiker_innen, sondern eine breitere Öffentlichkeit von Interesse sind, zeigte das Motto des 14. Bundeskongresses Politische Bildung 2019: „Was uns bewegt. Emotionen in Politik und Gesellschaft". ${ }^{22}$

\footnotetext{
1100-1800“ siehe URL: <http://www.historyofemotions.org.au/events/emotions-movement-culturalcontact-and-exchange-1100-1800/?page $=2>$ [Zugriff: 22.02.2019].

${ }^{21}$ Siehe Jarzebowski, Claudia: Kindheit und Emotion. Kinder und ihre Lebenswelten in der europäischen Frühen Neuzeit, De Gruyter, Berlin u. a. 2018; dies.: Tangendo. Überlegungen zur frühneuzeitlichen Sinnes- und Emotionsgeschichte, in: Brendecke, Arndt (Hrsg.): Praktiken der Frühen Neuzeit. Akteure, Handlungen, Artefakte (Frühneuzeit-Impulse, Bd. 3), Böhlau, Köln u. a. 2015, S. 391-404; Boquet, Damien/ Nagy, Piroska: Medieval Sensibilities. A History of Emotions in the Middle Ages, Polity Press, Cambridge 2018; Althoff, Gerd: Tränen und Freude. Was interessiert Mittelalter-Historiker an Emotionen, in: Frühmittelalterliche Studien 40 (2006), S. 1-11; Rosenwein/Cristiani: Emotions (wie Anm. 6); Boddice: History (wie Anm. 6).

${ }^{22}$ Die Programme zu den Konferenzen „Emotions and International Relations“ und „Public Emotions. Affective Collectivity in Audiences" sind unter der URL: <http://www.historyofemotions.org.au/media/ 178001/emotionsir-program-2014.pdf> [Zugriff: 11.03.2020] beziehungsweise unter der URL: <https:// conference.imp.fu-berlin.de/crc1171-public-emotions/home> [Zugriff 22.02.2019] abrufbar; Informationen zu den Tagungen „Emotionen, Politik und Medien in der Zeitgeschichte“ vom 17.10.2018-19.10.2018 an der Universität des Saarlandes, in: H-Soz-Kult, 29. August 2018, URL: <https://www.hsozkult.de/ event/id/termine-37989> [Zugriff 13.04.2019] und zu „Internationale Beziehungen und ,emotional regimes'. Neue Fragen an die Geschichte des Kalten Krieges“ unter URL: <https://www.hsozkult.de/event/ id/termine-36156> [Zugriff: 11.03.2020]; für Details zum Projekt zu Musik und Menschenrechten siehe URL: <https://blogs.fu-berlin.de/musicandhumanrights/> [Zugriff: 26.02.2019] und zum Bundeskongress Politische Bildung siehe URL: <https://www.bpb.de/veranstaltungen/format/kongress-tagung/269250/ 14-bundeskongress-politische-bildung-2019-was-uns-bewegt-emotionen-in-politik-und-gesellschaft> [Zugriff 22.02.2019]. Siehe auch Hitzer: Emotionsgeschichte (wie Anm. 6), S. 1 f., Anm. 3.
} 


\section{Emotionen in der Geschichte der internationalen Beziehungen}

Was also kann die Emotionsgeschichte mit Blick auf die Geschichte internationaler Beziehungen leisten? Emotionsgeschichte zeichnet Gefühlsartikulationen diachron nach, untersucht Spannungen zwischen Impulsen und sozialen Normen, bereichert Studien zu Themen wie race, class und gender und analysiert vor allen Dingen, inwiefern Emotionen politische Veränderungen, etwa bezüglich Nationalismus oder Immigration beeinflussten. ${ }^{23}$ So beleuchten eine Reihe von Aufsätzen unter dem Titel „Emotions in American History“ in transnationaler und transatlantischer Perspektive Themen wie Krieg, öffentliche Meinung, Kunst und die Medien mit besonderem Augenmerk auf Emotionen. Damit bieten sie eine spezifische Sichtweise auf Phasen des Übergangs in der US-amerikanischen Geschichte und korrelieren emotionalen Wandel mit wirtschaftlichen, gesellschaftlichen und strukturellen Veränderungen. ${ }^{24}$

Darüber hinaus haben eine Reihe von Autor_innen auf Zusammenhänge zwischen Emotion und Macht beziehungsweise politischen Entscheidungen hingewiesen. Als emotionale Wahlverwandtschaft (,elective emotional affinity“, S. 8) etwa beschreibt Gienow-Hecht das positive Verhältnis, das sich in der zweiten Hälfte des 19. Jahrhunderts zwischen US-Amerikanern und deutscher sinfonischer Musik entwickelte. Diese besondere Beziehung sollte zwei Weltkriege überdauern und wurde während des Kalten Krieges gezielt eingesetzt, um sich eines gemeinsamen kulturellen Erbes in Abgrenzung zu den kommunistischen Staaten zu versichern. ${ }^{25}$ Arbeiten zu den emotionalen Beweggründen für politische Entscheidungen aufseiten individueller Politiker haben die Historiker_innen Barbara Keys und Frank Costigliola vorgelegt. ${ }^{26}$ Keys analysiert die Freundschaft des hoch-emotionalen, nach außen sich kühl kalkulierend gebenden Henry Kissinger zum sowjetischen Botschafter Anatolij Dobrynin. Vermittels dieser Freundschaft und den damit einhergehenden Emotionen und Gewohnheiten, so Keys, lassen sich Kissingers politische Entscheidungen, unter anderem auch sein Festhalten am Bipolarismus trotz gegenläufiger politischer Realitäten, erklären. ${ }^{27}$ Um zu verstehen, wie es überhaupt zum Kalten Krieg kommen konnte, nimmt Costigliola Persönlichkeit, Beziehungen, Biografien, emotionale Dispositionen und die kulturellen Hintergründe von Schlüsselfiguren wie Theodore Roosevelt, Winston Churchill, Josef Stalin und Harry S. Truman in den Blick. Im diplomatischen Handeln innerhalb der Grand Alliance und der Nachkriegspolitik greifen für ihn Persönliches und Politisches ineinander. Die Parameter, die den je-

\footnotetext{
${ }^{23}$ Gienow-Hecht, Jessica C. E.: Introduction, in: dies. (Hrsg.): Emotions in American History. An International Assessment, Berghahn, Oxford/New York 2010, S. 1-14, hier S. 2 f.

24 Ebd., S. 4. Der Satz „Damit bieten sie eine spezifische Sichtweise auf Phasen des Übergangs in der USamerikanischen Geschichte und korrelieren emotionalen Wandel mit wirtschaftlichen, gesellschaftlichen und strukturellen Veränderungen." bezieht sich nicht auf den Inhalt des besprochenen Sammelbandes, sondern einen zuvor referierten Forschungsüberblick (ebd. S. 3).

25 Dies.: Sound Diplomacy. Music and Emotions in Transatlantic Relations, 1850-1920, Chicago UP, Chicago, IL 2009, S. 7 f., 15-18, 215.

26 Keys, Barbara: Henry Kissinger: The Emotional Statesman, in: Diplomatic History 35 (2011), H. 4, S. 58-609; Costigliola, Frank: Roosevelt's Lost Alliances. How Personal Politics Helped Start the Cold War, Princeton UP, Princeton, NJ/Oxford 2012; ders.: Reading for Emotion (wie Anm. 9).

27 Keys: Kissinger (wie Anm. 26), S. 594-609, hier S. 590, 602, 605, 609.
} 
weiligen emotionalen Glauben (,emotional belief“", S. 12) historischer Akteure einen logischen Sprung, im Zuge dessen das Faktenwissen an die Version der Realität, die man glauben möchte, angepasst wird - bedingen, können die Sichtweise und Ziele von Politikern erklären. ${ }^{28}$ In seinem Aufsatz „Reading for Emotion“ schließlich nimmt Costigliola deutlich zur Schau gestellten Ärger in den internationalen Beziehungen während des Kalten Krieges in den Blick. Er zeigt auf, inwiefern die „Lizenz“, Emotionen zu zeigen, proportional zur eigenen Machtfülle war und der Vorwurf, zu emotional zu sein, den Beschuldigten in seiner Macht einschränkte. Entsprechend folgert Costigliola, dass das Zurschaustellen und Reagieren auf solcherart gezeigte Emotionen Aufschluss über Machtverhältnisse geben können. ${ }^{29}$

\section{Nikita Chruschtschow, Saddam Hussein und die Logik des Affekts}

Wie Keys und Costigliola konzentriert sich auch Markwica auf historisch bedeutsame politische Führungsfiguren. Das von ihm vorgeschlagene Modell geht jedoch in seiner Systematik weiter und erhebt einen größeren Anspruch auf Transferierund Generalisierbarkeit, als das bei Keys und Costigliola der Fall ist. Emotion, so Markwica, ist nicht nur ein add-on, sondern eine zentrale Variable im politischen Handlungsprozess besonders in globalen Krisenmomenten. Mehr noch: Wenn sich Beobachter und Akteure des politischen Prozesses der Signifikanz dieser Variablen und ihrer historischen Manifestation bewusst seien, dann werde es in zukünftigen Szenarien denkbar und möglich sein, alternative Handlungsspielräume zur Friedenssicherung zu erschließen.

Potenzielle Reaktionen vorwegnehmend spricht sich der Autor gegen eine Lesart der im Untertitel genannten ,logic of affect“ als Oxymoron aus. Er bezieht sich bei der Entwicklung dieser Logik auf Erkenntnisse aus Neurologie und Psychologie, die Emotionen als Voraussetzungen für rationales Verhalten begreifen und einzelnen Emotionen bestimmte Tendenzen hinsichtlich der mit ihnen verknüpften Bewertung und Handlung zuschreiben. Diese laut Markwica nahezu universellen Tendenzen werden mit Variationen bedingenden Faktoren aus dem sozialen Umfeld und der persönlichen Disposition des zu untersuchenden Individuums kombiniert.

In einer ausführlichen Einleitung listet Markwica zunächst etablierte Erklärungsmodelle für die Konsequenzen von diplomatischer Zwangsausübung auf. Hierbei identifiziert er drei Lager: Rationalistische Ansätze gingen vom Homo oeconomicus aus, der autonom agiert und Informationen über mögliche Konsequenzen verschiedener Handlungsoptionen sammelt und evaluiert, um dann strategisch zu entscheiden. Von der Kognitionspsychologie inspirierte Arbeiten setzten dem Homooeconomicus-Modell entgegen, dass Menschen Signale und Bedrohungen nicht alle in gleicher Weise interpretieren. Vielmehr wirkten unter anderem kognitive Schemata auf die genannten Denkprozesse. Solche Schemata seien situative Schablonen,

\footnotetext{
28 Costigliola: Alliances (wie Anm. 26), S. 12 f. Das Konzept des emotional belief, auf das Costigliola Bezug nimmt, stammt aus Mercer, Jonathan: Emotional Beliefs, in: International Organization 64 (2010), H. 1, S. 1-31, hier S. 6.

29 Ders.: Reading for Emotion (wie Anm. 9), S. 366-369.
} 
die beeinflussen, wie Menschen neue Informationen interpretieren. Konstruktivistisch inspirierte Erklärungsansätze schließlich deuteten die entscheidende Person als Homo sociologicus. In ihren Entscheidungen sind Menschen demnach von je nach Kultur variierenden sozialen Normen beeinflusst. Im Falle einer diplomatischen Zwangssituation würden betroffene Akteure demnach nur kooperieren, wenn dies ihr jeweiliges kulturelles Skript zuließe. Diese drei Erklärungsmodelle, so stellt Markwica fest, klammern die Kategorie der Emotionen in Situationen der diplomatischen Zwangsausübung weitgehend aus. Ziel der Monografie sei daher, diese Forschungslücke zu schließen.

Im Anschluss an diese Einleitung stellt der Autor seine Logik des Affekts beziehungsweise „Emotional Choice Theory“ (ECT) vor, die als Ergänzung und Alternative zu eben jenen etablierten Erklärungsmodellen dienen soll, welche sich auf Konsequenzen und Angemessenheit konzentrieren. Die Theorie besagt, dass politische Führungspersonen, die sich einer Diplomatie der Zwangsausübung oder gar Bestrafung ausgesetzt sehen, nicht allein anhand von langfristiger Nutzenmaximierung und Identität agieren, sondern sich zudem an Emotionen orientieren. Anders als in der Auffassung der schwedischen Soziologin Helena Flam lässt Markwica keine Ausschließlichkeit und auch keine Hierarchie zu: Emotion fungiert hier als ein Handlungsantrieb, jedoch bilden Charakteristiken aller drei Agenten (Homo oeconomicus, Homo sociologicus, Homo emotionalis) eine Logik des Affektes oder eben auch ECT. Das Modell berücksichtigt neben der sozialen Dimension von Emotionen auch ihren körperlichen und dynamischen Charakter. Markwica definiert demnach Emotionen als teils biologisch, teils kulturell bedingte Reaktionen auf einen Stimulus, der zunächst einen Prozess bestehend aus Beurteilungen, Gefühlen, körperlichen Reaktionen und expressivem Verhalten auslöst. Menschen sind ihren Emotionen weder völlig ausgeliefert, noch können sie sie gänzlich kontrollieren. Die Grundthese ist, dass das Zusammenspiel von Identitäten, sozialen Normen und eben Emotionen - wie zum Beispiel Angst, Ärger, Hoffnung, Stolz und Demütigung - Entscheidungsprozesse nachhaltig beeinflussen kann.

In einem nächsten Schritt formuliert der Autor auf der Basis der Beurteilungsund Aktionstendenzen, die mit den fünf untersuchten Emotionen verknüpft sind, eine Reihe von Aussagen zu möglichen Verhaltensweisen von Politikern, die sich in einer außenpolitischen Zwangssituation befinden. Er betont hierbei, dass diese Prognosen, die das Herzstück seiner Logik des Affekts bilden, als Wahrscheinlichkeiten, nicht aber in deterministischem Sinne zu verstehen seien. Empfindet eine politische Führungsperson beispielweise angesichts eines drohenden militärischen Angriffs durch einen überlegenen Gegner primär Angst, bieten sich als mögliche Verhaltensweisen in Reaktion darauf Flucht in Form von Nachgeben, Widerstand oder eine Art Schockstarre an. Ein Einlenken der unter diplomatischer Bedrohung stehenden Politiker_innen ist unwahrscheinlich, wenn sie den Zwangsausübenden gegenüber Ärger empfinden, wenn die Politiker_innen Stolz über Handlungen empfinden, für die sie nun in der internationalen Arena zur Rechenschaft gezogen werden, oder wenn Hoffnung besteht, ungestraft davonzukommen.

Es ist dem Autor hoch anzurechnen, dass er sich im dritten Kapitel ausführlich mit der Herausforderung der Deduktion auseinandersetzt: Wie genau können wir rückblickend Emotionen und deren Einfluss erkennen? Markwica zieht zu diesem Zweck 
psychologische Techniken zur Ablesung von Emotionen insbesondere in schriftlichen Quellen heran und liefert darüber hinaus Anleitungen zur Kontextualisierung derselben zwecks deren Analyse im Kontext von Entscheidungsprozessen. Dabei ist er kein Idealist und führt selbst die Grenzen der Messbarkeit von Emotionen an: Die Lücke zwischen Gefühl und Darstellung; die Beschränkung von Text zuungunsten all dessen, was eine Person oder Zeugen nicht referieren; die Grenzen des Erfahrungsraumes der forschenden Person. Diese Ausführungen zur Methodologie liefern im Bereich der Emotionsgeschichte weniger erfahrenen Leser_innen durch die Hinweise auf Genres und rhetorische Strategien, die zur Affekt-Rekonstruktion herangezogen werden können, wertvolle Anhaltspunkte. Hinweise auf Audioaufnahmen und Stimmlagen zeigen weitere mögliche Recherchewege auf.

Im nächsten Schritt wendet der Autor das Modell der emotional choices auf zwei Fallstudien beziehungsweise zwei Fragestellungen an: Kapitel 4 widmet sich der Frage, warum Nikita Chruschtschow während der Kubakrise im Oktober 1962 in einer Situation des zunächst ausgeglichenen Kräfteverhältnisses nach nur mehr dreizehn Tagen die auf der karibischen Insel neu stationierten Mittelstreckenraketen wieder abzog und damit den Forderungen der USA entsprach. Kapitel 5 fragt, warum Saddam Hussein es während des ersten Golfkrieges 1991 ablehnte, seine Truppen aus Kuwait abzuziehen und sich damit willentlich und wissentlich gegen die Übermacht der Koalitionsstreitkräfte stellte. Dabei begründet Markwica seine Konzentration auf die Gefühle Chruschtschows und Husseins damit, auf diese Weise eine tiefergehende Analyse vornehmen zu können. Sein Ausklammern der Emotionen aufseiten der Gegenpartei, in diesem Fall amerikanischer Politiker, soll nicht das Stereotyp des rationalen Westens in Opposition zum emotionsgesteuerten Osten reproduzieren. John F. Kennedy und George H. W. Bush waren ebenfalls stark emotional involviert, was Markwica an entscheidenden Stellen berücksichtigt.

Diese beiden Fälle sind auf den ersten Blick nicht vergleichbar; Saddam Hussein mag ein regional gefürchtetes Staatsoberhaupt gewesen sein, nicht aber ein globaler Hegemon wie Chruschtschow und auch der Irak lässt sich beim besten Willen nicht mit dem imperialen Anspruch der Sowjetunion vergleichen. Strategisch jedoch stellen beide Fälle ein Beispiel der US-amerikanischen Diplomatie der Zwangsausübung dar. Diese funktionierte im Falle der Kubakrise ganz offensichtlich: Chruschtschow erkannte die Ernsthaftigkeit der US-Bedrohung und ließ sich dementsprechend auf einen Kompromiss ein, der ihm erlaubte, Konzessionen (Abzug der US-Jupiterraketen aus der Türkei) zu erwirken. Im zweiten Fall funktionierte die Diplomatie der Zwangsausübung der USA nicht: Bis zum Schluss ignorierte der irakische Führer sowohl die Ratschläge seiner eigenen Berater als auch die militärische Übermacht der Koalitionskräfte.

Warum führte eine scheinbar gleiche politische Strategie zu unterschiedlichen Ergebnissen? Markwica führt zur Beantwortung dieser Frage eine Tiefenanalyse der in Äußerungen, Augenzeugenberichten sowie Egodokumenten nachweisbaren Emotionsausdrücke durch, um am Ende eine Tabelle individueller Entscheidungsschritte und der dabei mitspielenden Emotionen und deren Relevanz aufzustellen.

Im Falle Kubas, so der Autor, standen im Vordergrund Chruschtschows Angst vor einer atomaren Eskalation gekoppelt mit Stolz beziehungsweise der Notwendigkeit zur hegemoniellen Anerkennung. Kennedys Diplomatie der Hintertür zeigte dem 
sowjetischen Staatsoberhaupt, dass der US-Präsident seine Stellung wertschätzte und einen Unterschied zwischen öffentlichen Aussagen und tatsächlicher Handlung machte; beides ermöglichte es dem sowjetischen Regierungschef, einen Rückzieher in der Karibik zu machen, ohne sein Gesicht zu verlieren.

Markwica arbeitet bezüglich Chruschtschows Emotionen auch die Schlüsselfunktion einer Mediatorenfigur heraus, nämlich die des sowjetischen Botschafters Anatolij Dobrynin. Per Telegramm informierte Dobrynin Chruschtschow über die Kampfbereitschaft des Pentagons, was wiederum Chruschtschows Angst verstärkte und ihn dazu bewog, Kennedy einen Handel vorzuschlagen - die sowjetischen Raketen würden abgezogen, wenn im Gegenzug die USA versprächen, Kuba nicht anzugreifen. Und es war wiederum Dobrynin, der Chruschtschow durch gewählte Formulierungen vermittelte, dass Kennedy bei der Durchsetzung dieses Kompromisses seine Hilfe benötigte und ihn als das Oberhaupt einer den USA ebenbürtigen Supermacht ansah. Diese Respektsbekundung habe bei Chruschtschow Ärger und Demütigung zugunsten von Selbstwert schwinden lassen und ihm ermöglicht, die amerikanischen Bedingungen ohne Gesichtsverlust anzuerkennen.

Ganz anders war die Sachlage im Falle des Golfkrieges: Die Bush-Administration lehnte von vornherein jegliche direkte Kommunikation mit dem irakischen Präsidenten und damit ein Verständnis für dessen Perspektive ab. Saddam Hussein wiederum zeigte ausgeprägte Gefühle des Stolzes gekoppelt mit Narzissmus und Realitätsverlust und legte noch im Angesicht totaler Annihilation Zuversicht und Siegerlaune an den Tag. Auch hier nimmt Markwica acht ausgewählte Entscheidungen Husseins unter die Lupe. Er analysiert dessen Verhalten vom Entschluss zur formalen Annektierung Kuwaits am 8. August 1990 bis zum am 25. Februar 1991 beschlossenen Abzug der irakischen Truppen. Markwica zeigt hier, dass Hussein sich dem Druck der USA, seine Soldaten aus Kuwait abzuziehen, zunächst widersetzte, da diese Eroberung seinem Stolz schmeichelte. Der irakische Präsident habe zudem Hoffnungen gehegt, die Amerikaner abschrecken zu können.

Neben diesen Emotionen beeinflussten Husseins Identität, sein kultureller Hintergrund und seine Emotionsnormen sein Verhalten entscheidend. Mit seiner emotionalen Sozialisierung und der Selbstkonzeption als arabischer Ritter (,,knight of the Arabs““, S. 180) war das Gefühl der Angst nicht vereinbar. Damit erklärt Markwica, weshalb Hussein, anders als seine Berater, zunächst keine Angst zeigte und sie, um nicht von ihrer Furcht angesteckt zu werden, dazu anhielt, ihre Bedenken für sich zu behalten.

Im Januar 1991 widersetzte sich Saddam Hussein nach wie vor dem von den Vereinten Nationen auf den 15. des Monats festgesetzten Ultimatum zum Abzug seiner Truppen. Seine Hoffnung sei nun gewesen, den übermächtigen Gegner mit der Hilfe Verbündeter doch noch zu schlagen. Dass er seine Angst weiterhin herunterspielte und, da er sich von der amerikanischen Regierung degradiert und in seiner Rolle als ,arabischer Ritter" nicht anerkannt sah, zunehmend Wut und Demütigung empfand, erkläre seine Entscheidung zur Konfrontation. Angesichts der Nachrichten über Iraks militärische Verluste hätten Angst und Demütigung Hussein schlussendlich dazu veranlasst, am 25. Februar 1991 den Befehl zum Abzug der Truppen zu geben. 
In beiden Fallstudien kommt der Autor zu dem Ergebnis, dass die Logik des Affekts nicht jede Verhaltensweise und Entscheidung der beiden Staatsmänner erklären kann. So sei im Falle Chruschtschows auch der Homo rationalis am Werk, denn seine Entscheidung, am 23. Oktober 1962 den Befehl zur Umkehr der bewaffneten Schiffe zu geben, sei nicht von Angst begleitet gewesen, weshalb sie Markwica als Ergebnis einer rationalen Kosten-Nutzen-Analyse einstuft. Auch der Entschluss am 27. Oktober 1962, den Abzug amerikanischer Raketen aus der Türkei zur Bedingung zu machen, lässt sich für Markwica nicht mit der Logik des Affekts begründen, da die verfügbaren Quellen keine entsprechenden Hinweise lieferten. Entsprechend vermutet der Autor, dass Chruschtschow hier seinen Vorteil suchte und als Homo oeconomicus agierte. Auch Saddam Husseins Verhalten lässt sich nicht ausschließlich auf der Basis seiner Emotionen erklären. Am 23. Februar 1991 machte er das Angebot, seine Truppen aus Kuwait abzuziehen, sofern alle den Irak betreffenden UN-Resolutionen widerrufen würden. In diesem Kontext macht Markwica in den Quellen keine Hinweise auf Emotionen aus und folgert daher, dass Hussein hier die Konsequenzen abgewogen und befunden habe, angesichts der militärischen Überlegenheit der USA Verluste zu minimieren und seine Machtposition zu sichern.

Am Ende schließt Markwica mit einem Plädoyer. Die Theorie der emotional choice sei eine Anleitung zukünftiger politischer Handlungsmöglichkeiten im Kontext der Diplomatie der Zwangsausübung. Reiner Zwang treibe den Gegner in eine Ecke, aus der er nur durch Selbsterniedrigung wieder herauskomme. Gerade dieser Schritt falle Führungspersönlichkeiten jedoch besonders schwer, weil sie Bedrohung häufig durch Realitätsverlust kompensierten. Dem folgt eine unmittelbare Handlungsempfehlung: Parteien, die Zwang auf eine politische Führungsfigur ausüben wollen, sollten bei der Formulierung und Kommunikationsform ihrer Warnungen die sozioemotionale Disposition der Empfänger abwägen, um zu vermeiden, dass diese Art der Kommunikation, etwa in Form einer öffentlichen Drohung, negative Effekte verstärkt. Markwicas Fallstudien implizieren zudem, dass ein Androhen physischer Attacken ganz unterschiedliche Emotionen und Verhaltensweisen hervorrufen kann. Auch Versprechen von Vorteilen im Falle einer Kooperation mit der überlegenen Seite seien unter Berücksichtigung der Emotionen vorsichtig zu formulieren. Respekt und ein Verzicht auf Beleidigungen und Degradierung seien hier ebenso wichtig wie das Inaussichtstellen materieller Gewinne. Was die zeitliche Abfolge der Formulierung von Drohungen und Versprechen im Falle eines Einlenkens angeht, so rät Markwica, dass Drohung und Versprechen nicht nacheinander, sondern zugleich formuliert am effektivsten zur Deeskalation beitragen könnten. Da die Reaktionen auf Drohungen auch stark von der Identität und von sozialen Normen beeinflusst werden, sollten Entscheidungsträger bei der Entwicklung von Strategien zur Zwangsausübung den kulturellen Hintergrund und das Identitätskonzept der Person, und die damit verknüpften Vorteile und Risiken im Falle einer Bedrohung abwägen. Von einer Vortäuschung kaum zu kontrollierender Emotionen im Zuge der Bedrohung sollten politische Akteure jedoch grundsätzlich absehen, da dies aufseiten der Bedrohten zu Misstrauen führen kann. Des Weiteren sollten Strategen, um diplomatische Zwangsausübung erfolgreich zu gestalten, Empathie zeigen und das Potenzial direkter, vertraulicher Kommunikation nutzen, zudem Mediator_innen 
heranziehen und dem Gegenüber die Möglichkeit einräumen, seine Sorgen und Beschwerden zu artikulieren.

„Emotional Choices“ ist eine Bereicherung der Literatur zum Wechselspiel von Emotion und internationalen Beziehungen: Erstens kombiniert Markwicas Untersuchung zwei unterschiedliche Forschungsstränge, die in der Vergangenheit eher nebeneinander als miteinander betrachtet wurden. Seine Arbeit unterstreicht die Kritik vieler Sozialwissenschaftler und Psychologen, die den Vertretern der rationalen Entscheidungstheorie übermäßige Emphase auf dem Handeln einzelner Individuen (great men, great history) sowie Indifferenz in Bezug auf Gesetzmäßigkeiten und Strukturorientierung vorgeworfen hat. Auch berührt das Werk die Diskussion um den Stellenwert von Emotionen im Kontext von Führungspersönlichkeiten in der internationalen Arena.

Zweitens inspiriert vor allem das Methodenkapitel in der Emotionsgeschichte wenig erfahrene Leser_innen, insbesondere durch das Benennen verschiedener Quellen-Genres und rhetorischer Strategien, die Aufschluss über Affekte geben können. Markwica selbst zieht autobiografische Dokumente, Selbsteinschätzungen, Augenzeugenberichte und biografische Studien heran. Anregend bezüglich der Erforschung historischer Affekte ist auch sein Hinweis auf nicht textgebundene Quellen. Videound Audioaufnahmen, so zeigt Markwica auf, erlauben eine Berücksichtigung paraverbaler Parameter wie Stimmhöhe oder nicht-verbaler Ausdrucksmittel wie Körpersprache.

Drittens formuliert Markwica seine Überlegungen flüssig und bietet auch einer im Fachgebiet weniger erfahrenen Leserschaft Orientierung, ohne Gefahr zu laufen, die jeweiligen Sachverhalte durch Vereinfachung unpräzise darzustellen. Klar strukturierte Teilkapitel und ein ebenso gestaltetes Inhaltsverzeichnis erlauben rasche Orientierung. Idealtypen, schematisierende Grafiken zu den fünf Schlüsselemotionen und Tabellen dienen der Veranschaulichung. Sie werden, potenzielle Kritik vorwegnehmend, ausbalanciert durch Anmerkungen zu Problemlagen, die durch Vereinfachung entstehen können. Dazu zählt auch der Hinweis auf die potenziellen Fallstricke, die eine Generalisierung des entwickelten Modells bergen kann - der Autor rät hier, stets die Vergleichbarkeit der gewählten Fälle im Blick zu behalten.

Einige Unstimmigkeiten bleiben. Der innovative Charakter der vorgestellten Methodik erschließt sich stellenweise nicht. Es wird nicht deutlich, inwiefern sich die vorgeschlagenen Analyseverfahren für in Schriftform verfügbare Quellen von etablierten Strategien wie Rhetorik- oder Diskursanalyse unterscheiden. Das komplexe Bündel an Faktoren, das es bei dem Versuch, eine spezifische Emotion zu erklären, zu berücksichtigen gilt, wirft Fragen nach der Praktikabilität des Ansatzes auf.

In Einzelfällen ließen sich in der Darstellung zudem Wiederholungen und Redundanzen vermeiden. In Bezug auf die beiden Fallstudien irritiert, dass der empirische Teil im Gegensatz zu den theoretischen Überlegungen die möglichen Schwierigkeiten, die aus Sprachbarrieren und Übersetzungen erwachsen können, offenbar unbeachtet lässt. Ob die zum Ende aufgelisteten Handreichungen hinsichtlich politischer Entscheidungsfindungen tatsächlich zu einer Veränderung diplomatischer Praktiken führen, ist fraglich. In ihrem Anliegen, zur Konfliktprävention beizutragen, sind sie jedoch plausibel. 
„Emotional Choices“ unterstreicht - ohne es zu erwähnen - jene postkolonialen Wissenschaftler wie Damcelle Torres-Cortes, die dezidiert zwischen kulturellen Verhandlungspraktiken im globalen Vergleich unterscheidet. ${ }^{30}$ Nichtsdestotrotz: Markwicas Buch hat das Potenzial, die Diskussion um Entscheidungsfindungsprozesse nachhaltig anzuregen. Es ist nicht allein seine historisch inspirierte Analyse, vielmehr sein schwerpunktmäßiges Augenmerk auf Emotion als einer weiteren Variable in diesen Prozessen, die relative Offenheit, Pluralität und Flexibilität des Ansatzes, die dem Buch einen besonderen Reiz nicht nur für die Sozialwissenschaften, sondern darüber hinaus für politische Beobachter und Entscheidungsträger selbst verleiht.

\section{Besprochene Literatur}

Markwica, Robin: Emotional Choices. How the Logic of Affect Shapes Coercive Diplomacy, 384 S., Oxford UP, Oxford u. a. 2018.

Funding Open Access funding provided by Projekt DEAL.

Open Access Dieser Artikel wird unter der Creative Commons Namensnennung 4.0 International Lizenz veröffentlicht, welche die Nutzung, Vervielfältigung, Bearbeitung, Verbreitung und Wiedergabe in jeglichem Medium und Format erlaubt, sofern Sie den/die ursprünglichen Autor(en) und die Quelle ordnungsgemäß nennen, einen Link zur Creative Commons Lizenz beifügen und angeben, ob Änderungen vorgenommen wurden.

Die in diesem Artikel enthaltenen Bilder und sonstiges Drittmaterial unterliegen ebenfalls der genannten Creative Commons Lizenz, sofern sich aus der Abbildungslegende nichts anderes ergibt. Sofern das betreffende Material nicht unter der genannten Creative Commons Lizenz steht und die betreffende Handlung nicht nach gesetzlichen Vorschriften erlaubt ist, ist für die oben aufgeführten Weiterverwendungen des Materials die Einwilligung des jeweiligen Rechteinhabers einzuholen.

Weitere Details zur Lizenz entnehmen Sie bitte der Lizenzinformation auf http://creativecommons.org/ licenses/by/4.0/deed.de.

Anna Rauscher ist seit 2018 Doktorandin im Fachbereich Geschichte am John-F.-Kennedy-Institut für Nordamerikastudien der Freien Universität Berlin. In ihrer Arbeit untersucht sie das Zusammenwirken von klassischer Musik und Menschenrechtsdiskursen in den USA in der zweiten Hälfte des 20. Jahrhunderts.

Jessica Gienow-Hecht ist Professorin für Neuere Geschichte und Direktorin des John-F.-Kennedy-Instituts für Nordamerikastudien an der Freien Universität Berlin. Ihre Forschungsschwerpunkte liegen im Bereich der internationalen und globalen Geschichte, der Geschichte der transatlantischen Beziehungen und der Relationen zwischen Nordamerika und Asien, dem Verhältnis zwischen (geo-)politischer Legitimierung und kulturellen Projektionen, sowie der Funktionen von nation branding, Musik, Emotionen und Humanitarismus in den internationalen Beziehungen.

\footnotetext{
30 Torres-Cortes, Damcelle: Glass Half Empty or Half Full? The AICHR and the ASEAN Perspective on Humanitarian Intervention, in: An Occasional Supplement to the Journal of Global Studies/Asian Perspectives on Humanitarian Interventions in the 21st Century (2013), S. 147-174, URL: <https://globalstudies.doshisha.ac.jp/attach/page/GLOBAL_STUDIES-PAGE-EN-73/80564/file/OS2013_9.pdf> [Zugriff: 20.09.2019].
} 